\title{
Fundación del Sistema de la Reserva Federal en Estados Unidos
}

Foundation of the United States Federal Reserve System

\author{
Mario Rojas Miranda* "
}

\section{Resumen}

El trabajo analiza la fundación del Sistema de la Reserva Federal en Estados Unidos en 1913 para resaltar que esta institución pretendía dotar de una moneda elástica y de movilidad de reservas que hiciera frente a las quiebras bancarias que se extendieron en todo el territorio durante el siglo xIX y principios del $\mathrm{xx}$, suscitando pánicos financieros que empujaban a la economía a una crisis. La creación del Sistema de la Reserva Federal representó el tercer intento por contar con un banco central. The First Bank en 1791 y The Second Bank en 1816 fueron sus antecedentes, los cuales evidentemente no lograron sobrevivir. Casi 100 años después, se presentó una nueva oportunidad, de la cual emergieron 12 bancos regionales que hoy día conforman la maquina monetaria de Estados Unidos.

Palabras clave:

- Sistema de la Reserva Federal

- Política monetaria

- Banca central

\begin{abstract}
This work analyzes the foundation of the United States Federal Reserve System in the year 1913 in order to highlight that this institution claimed to provide an elastic currency and the mobility of the reserves that would address bank failures throughout the country during the 19th and early 20th century, raising financial panic that pushed the economy into a crisis. The creation of the Federal Reserve System represents the third attempt to have a central bank. The First Bank, in 1791, and The Second Bank, in 1816, were its antecedents, neither of which, obviously, have survived. Almost 100 years later, a new opportunity presents itself: the emergence of 12 regional banks that today conform to the monetary model of the United States.
\end{abstract}

JEL: E42, E52, E58

\section{Presentación}

El 13 de diciembre de 2013 se cumplen 100 años de la creación del Sistema de la Reserva Federal (Fed), que es el banco central de la principal economía del mundo. La producción de Estados Unidos (EU) representa 22\% de la economía mundial y su instituto central es la maquinaria monetaria más influyente del planeta. A un siglo de su existencia es considerado como una figura insignia de su poder económico. En 1913 hubo entre los líderes políticos el consenso necesario sobre la urgencia de contar con una banca central. Sin embargo, su creación no fue una tarea sencilla, se trató de un proceso intenso de negociación que satisfizo posiciones de izquierda, centro y derecha, debido a que éstos tenían más de una visión de cómo debía ser. Al final se trató de un experimento único.

\footnotetext{
* Estudiante del Doctorado en Economía en la División de Estudios de Posgrado de la UNAM . • •
} 
Con la fundación del Fed, la mayoría de los países del mundo comenzaron a crear sus propios bancos centrales. En México, los gobiernos post-revolucionarios fundaron el Banco de México en 1925, era de esperarse que, la fundación del Fed de 1913 influyera a los legisladores en 1917. Desde hace 100 años, el Fed ha atestiguado significativos sucesos económicos tales como: La Gran Depresión de la década de 1930, la Gran Inflación de la década de 1950, La Gran Moderación que inicio en 1980 y la Gran Recesión que aún aqueja al mundo. La existencia de los bancos centrales todavía es motivo de un gran debate. Algunos economistas resaltan su trabajo para estabilizar la inflación en torno a un blanco y el producto en torno al producto potencial. Otros, por el contrario, sostienen que los bancos centrales son responsables de las oscilaciones del producto y de crear, mal administrar y agudizar las crisis económicas.

El trabajo sostiene que la creación del Fed en 1913 se debió a la necesidad que tenía Eu de dotar a su economía de una moneda elástica y de movilidad de reservas que hiciera frente a las quiebras bancarias que se extendieron en todo el territorio, en el siglo XIX y principios del xx, suscitando pánicos financieros que se generalizaban, poniendo en riesgo el sistema bancario y financiero y arrojando a la economía a una crisis. La creación del Fed representó el tercer intento por contar con un banco central. The First Bank en 1791 y The Second Bank en 1816 fueron sus antecedentes, éstos no lograron sobrevivir al entorno económico y político de la época. El siglo que siguió atestiguó el desarrollo del sistema bancario y financiero y de los pánicos financieros, en particular en 1907, que en ausencia de una institución gubernamental que le hiciera frente, la contingencia fue asistida por J. P. Morgan, el banquero más influyente del mundo, urgiendo al gobierno a crear un banco central.

El trabajo se divide en dos apartados, el primero aborda tres aspectos relevantes: i) The Fist Bank of the United States el primer banco nacional que surgió en 1791 después de que las trece colonias alcanzaron su independencia y su extinción después de un lapso de veinte años, ii) The Second Bank of the United States como un banco que alcanzó a tener 25 sucursales y solucionó muchos de los problemas que se heredaron de la guerra con Inglaterra y iii) el pánico financiero de 1907 que despertó el interés del gobierno por crear un banco central. En la segunda parte se aborda la creación del Fed desde la presentación de los planes Aldrich y Valderlip, las dificultades para su aprobación y los desafíos que enfrentó la Comisión para elegir las ciudades sedes de los 12 bancos y el trazo de los distritos que conforman el Fed. Al final se presentan las conclusiones. 
Economía Informa núm. 385 marzo - abril • 2014 - "

\section{El primer y segundo intento, y el pánico financiero de 1907}

Hacia el último cuarto del siglo XviII, las trece colonias británicas enfrentaron una batalla con Inglaterra, logrando su independencia en 1783. Como una nación joven, EU enfrentó amenazas de desintegración, endeudamiento y una inflación galopante. Alexander Hamilton, primer secretario del Tesoro, encabezó los esfuerzos para crear The First Nacional Bank (FB), sus referentes analíticos fueron las obras de grandes economistas como: Adam Smith, David Ricardo y David Hume y en la práctica el Banco de Inglaterra, el cual era un banco de emisión totalmente privado y orgullo del imperio británico. $\mathrm{Su}$ creación enfrentó la oposición de Thomas Jefferson, Secretario de Estado, quien temía que un banco nacional pudiera constituir un monopolio financiero que atentara contra la dominancia de los bancos estatales.

El FB fue creado en 1791 para operar por 20 años y la posibilidad de ser ratificado para periodos iguales posteriores, se constituyó con 10 millones de dólares, el gobierno aportó 20 y 80\% restante fue inversión privada. Éste comenzó a operar en Philadelphia hasta alcanzar 8 sucursales, se conformó con una junta de 25 directores para supervisar sus operaciones, de los cuales 5 fueron nombrados por el gobierno y el resto elegidos entre los bancos privados. Las actividades abarcaron prácticas públicas y privadas tales como: recaudador de impuestos, depositario de los fondos públicos, prestamista del gobierno, de estados, autoridades locales y extranjeros; facilitó la trasferencia de recursos públicos a través de la red de sus sucursales y administró el pago de intereses de la deuda en manos de extranjeros, entre otros (Sylla et al, 2009).

Las sucursales del FB, establecidas en lugares de alta concentración económica facilitaron el préstamo a los particulares estimulando el comercio dentro y fuera de EU. El pago de impuestos federales era realizado mediante el uso de los billetes del FB, también llamadas notas bancarias, mismas que coexistieron con las de los bancos estales. Las notas del FB recorrieron el Este, el Centro y el Oeste de Eu. La capitalización del banco nacional se realizó mediante una oferta de títulos al público inversionista nacional e internacional, que dada la enorme aceptación, provocó una burbuja financiera, inestabilidad bancaria, quiebra de bancos y zozobra en el mercado financiero. La ola especulativa provocó una baja en los niveles de crédito, afectando el consumo, la inversión y la economía real.

En respuesta, Hamilton comandó operaciones de mercado abierto por parte del FB con la intención de suministrar liquidez al sistema bancario y financiero y apaciguar la crisis económica. El banco nacional actuó como un 
banco central en la era moderna, en los hechos Hamilton se había conducido como el primer banquero central de EU Sin embargo, el desempeño del FB no fue bien recibido por todos. Sus críticos resaltaron que el alivio había sido temporal y que estas acciones coincidieron con el surgimiento de una nueva burbuja financiera aunque de menor intensidad, sobre todo se comenzó a relacionar a esta gran institución con quiebras y problemas financieros, pensaban que la avaricia del dinero generaba más males que beneficios y que su permanencia ponía en riesgo a la economía. Al cumplir el banco su aniversario numero veinte, los miembros del congreso decidieron no renovar su permiso y el FB se liquidó (Federal Reserve Bank of Philadelphia, 2009).

En 1812 el país entró en guerra contra Inglaterra misma que concluyó dos años después. La batalla bélica desquició las actividades productivas, la deuda pública se incrementó y la economía experimentó un alza de precios. El gobierno tuvo necesidades crecientes de recursos, la guerra había tenido costos muy altos y las fuentes de financiamiento internas y externas eran escasas. Desde la extinción del FB las finanzas públicas se habían deteriorado considerablemente, las oficinas de recaudación de impuestos del gobierno habían cerrado con la liquidación del primer banco nacional. Los estados contribuyeron con muy poco para financiar la batalla contra uno de los imperios más poderosos de Europa Occidental, los gastos fueron absorbidos mayoritariamente por la federación, quien había enfrentado la contingencia en las peores condiciones financiera.

Con la ausencia de una banca nacional, la proliferación de bancos en los estados con potestad de emitir moneda, en muchos casos de manera desquiciada, habían alentando desordenes monetarios. El contexto económico y político para crear un banco nacional era propicio y fue aprovechado. El entonces presidente Madison atestiguó la creación de The Second Bank of the United States, también llamado The Second Bank (sB), el cual comenzó operaciones en Filadelfia en 1917, en la misma ciudad donde inicio el FB, hasta alcanzar 25 sucursales. El sB tuvo las mismas características y atribuciones que el FB, en realidad se trató de una copia, con la diferencia de que su capital inicial fue de 35 millones de dólares, lo cual lo hizo ser en ese momento el banco más grande e influyente en el ámbito financiero no solo de eu, sino de toda América.

A diferencia de los bancos centrales modernos de hoy en día, el FB y el sB no fijaron oficialmente la política monetaria y tampoco regularon a los bancos. Sin embargo, su tamaño, su capitalización, su credibilidad y la posición geográfica de sus sucursales les permitieron conducir una política monetaria "rudimentaria", debido a que las políticas de préstamo y de flujo de fondos del 
Economía Informa núm. 385 marzo - abril • 2014 - "

FB y del SB alteraban constantemente la oferta de dinero, el crédito y el tipo de interés para los prestatarios o usuarios del crédito. Además, los billetes del banco, respaldados por reservas de oro y plata sustanciales le permitieron al país contar con una moneda nacional, que además de ser estable, facilitó el intercambio comercial. Sin lugar a dudas, estas son algunas acciones de política monetaria que tienen vigencia y efectos hoy en día.

El desempeño del gigante financiero había atraído nuevamente la atención de muchos, principalmente de pequeños propietarios, comerciantes e industriales, quienes veían en esta institución una amenaza para sus negocios. Creían que los grandes intereses bancarios y financieros podían llegar a dominarlo todo, incluyendo a ellos. Este sentimiento se volcó al espectro político y fue capitalizado por algunos representantes entre ellos, Andrew Jackson, candidato a la presidencia por el Partido Demócrata, quien tomó como bandera el rechazo de la segunda banca nacional, resultando electo en 1828. Jackson vio su triunfo como una prueba de que la gente no deseaba el SB. Al igual que en el primer caso, el banco no contó con la fuerza suficiente de los legisladores y no logró su renovación (Federal Reserve Bank of Philadelphia, 2010).

Los pánicos financieros y las corridas bancarias fueron comunes a lo largo del siglo XIX y los primeros años del XX. Algunas de estas crisis fueron más severas que otras pero la mayoría siguió el mismo patrón. Todo comenzaba con un rumor sobre el colapso de un banco, ésto minaba la confianza sobre el conjunto del sistema bancario y el público se volcaba a hacer filas en los bancos. El miedo de los depositantes a perderlo todo generaba una oleada de retiros. Sin fondos líquidos para atender la demanda, en un lapso breve los bancos estaban al borde de la quiebra, muchos no lograban sobrevivir y el contagio se expandía. En respuesta el crédito se restringía. El verdadero problema recaía en la inelasticidad de la moneda y la falta de movilidad de las reservas bancarias que se manifestaban con las crisis y se trasladaban al resto de la economía.

El pánico financiero que ha recibido mayor atención en la literatura económica ha sido indiscutiblemente el de 1907, no por la severidad de sus secuelas en la sociedad sino porque generó un ambiente de reflexión. Cuando el pánico financiero se suscitó, John Pierpont Morgan, la figura dominante de la banca en América, encabezó los esfuerzos para paliar la crisis, designando un comité de banqueros que tendrían como tarea auditar a las instituciones bancarias con problemas y determinar sus necesidades de capitalización, George Cortelyou, entonces Secretario del Tesoro, se trasladó a Nueva York para acordar acciones conjuntas de apoyo con Morgan. Es importante destacar 
que Morgan no viajó a Washington para entrevistarse con el representante del Tesoro, sino que el Secretario se dirigió a Nueva York para encontrarse con Morgan (Tallman y Moen, 1990).

La crisis cedió y el presidente Roosevelt agradeció públicamente, con sus palabras: "la admirable forma en la cual ustedes (Morgan y otros banqueros) habían manejado la presente crisis" y aunque el primer mandatario nunca mencionó a Morgan por su nombre, señaló “aquellos hombres de negocios considerables y conservadores quienes han actuado en esta crisis con sabiduría y espíritu público". Morgan había hecho las funciones que comúnmente realiza un banquero central, es decir, generar confianza entre el público de que la contingencia será atendida suministrando liquidez al sistema, evitando que el crédito se interrumpa y que no contamine a los distintos sectores de la economía real. Cabe destacar que ésta no fue la primera ocasión que una contingencia financiera era atendida por Morgan ante la ausencia de una banca central (Federal Reserve Bank of Boston, 2011).

¿Cuál era el problema? Con el paso de los años eu desarrolló un sistema bancario en donde los préstamos y las actividades de inversión de las instituciones estaban ligados con la creación de depósitos. En 1867 por cada 1.2 dólares de depósitos existía 1 dólar en moneda en circulación, esta relación se fue modificando en los siguientes años, incluso décadas. En 1872 la relación era de 2/1, para 1879 de 4/1, 1898 de 6/1 y de 9/1 cuando se desencadenó el pánico financiero de 1907. Es por ello que, cualquier dificultad bancaria que se generalizaba debilitaba la confianza del público en los bancos provocando una caída en la relación de los depósitos a la moneda, porque el público buscaba convertir en muy poco tiempo sus depósitos en monedas. El sistema bancario era altamente vulnerable a cambios bruscos en las preferencias de liquidez de la gente.

Esta alta relación de depósitos a moneda hizo a los bancos más susceptibles de quebrar a través de un pánico, generando que los bancos tuvieran un fuerte incentivo en reforzar sus posiciones de efectivo al más ligero signo de retiro de depósitos. Todo intento desordenado de parte del público por convertir sus depósitos en dinero drenaba las reservas de los bancos. En un sistema bancario unitario con más de 20 mil bancos independientes el impacto era desigual, forzando algunos bancos a la suspensión y amenazando una reacción en cadena por el deseo del púbico para convertir sus depósitos en moneda. La mayoría de las veces la respuesta fue la restricción de pagos. Esta medida protegió el sistema bancario y dio tiempo para que el pánico pasara, así como para que la moneda estuviera disponible y la restricción de pagos se levantaba cuando la probabilidad del daño disminuía (Friedman y Schwartz, 1963). 
Economía Informa núm. 385 marzo - abril • 2014 - "

\section{Los planes Adrich y Valderlip: el tercer intento}

El ambiente de crisis abrió el camino para la discusión de una nueva reforma al sistema bancario y financiero que resolviera los problemas de inelasticidad de la moneda y la inamovilidad de las reservas bancarias. En 1908 el Congreso creó la Comisión Monetaria encargada de elaborar un estudio sobre los cambios al sistema bancario y financiero, además se incluyó una reforma financiera para lograr, aunque con limitaciones, hacer que la oferta de dinero fuera más elástica en momentos de emergencia. La Comisión estuvo liderada por el senador republicano Nelson W. Aldrich de Rhode Island, el más poderoso miembro del Senado y representante del poder económico del Este del país. La comisión recibió la venia de los banqueros y hombres de negocios y el rechazó de los pequeños productores del campo, industriales y comerciantes, entre otros.

Después de tres años, en 1911 el senador Aldrich expuso en Washington ante un grupo de banqueros y hombres de negocios su plan para reformar las instituciones del sector financiero, que sería llamado el Plan Aldrich. Evidentemente el proyecto contempló las aspiraciones de los hombres más influyentes de Eu sobre cómo debía ser un banco central, el cual sería llamado National Reserve Association con sucursales en todo el país, con el poder de emitir dinero y re-descontar el papel comercial de los bancos miembros, su administración y control residiría en una junta de directores, la cual estarían conformada en su mayoría por banqueros prominentes. En síntesis, se trató de un plan en donde la nueva estructura financiera y bancaria estaría bajo la administración de un instituto centralizado y bajo el control absoluto de la iniciativa privada.

Sin embrago, la aprobación de la propuesta se vio interrumpida por las elecciones presidenciales. El partido republicano enarbolaba los intereses de las familias más ricas de eu y tenía sus bases de apoyo en la parte Este del país. Por su parte, el partido demócrata exaltaba las demandas del movimiento progresista, - formado por pequeños productores y la clase baja-, el cual buscaba limitar el poder de la comunidad financiera y tenía sus bases en el Sur y el Centro del país. Estaba claro que los republicanos apoyaban el Plan Aldrich y los demócratas, cercanos al movimiento progresista, lo rechazaban. Los temas financieros ocuparon un lugar destacado en la riña electoral, el partido republicano perdió las elecciones de 1913 y el demócrata Woodrow Wilson se alzó con la victoria y nombró a Willian Jennings Bryan, quien fuera enemigo número uno del Plan Aldrich, como su secretario de Estado (Moen y Tallam, 1999). 
Estaba claro que el Plan Aldrich sufriría cambios. Bajo el mandato de Wilson, se nombró a Carter Glass, demócrata de Virginia, como jefe de la Comisión de Banca y Finanzas encargada de dictaminar el nuevo instituto central. Glass presentó su nueva propuesta de Plan Aldrich que contempló: la creación de 20 o más bancos regionales controlados en su mayoría por los privados, los cuales tendrían una porción de las reservas de los bancos miembros, desarrollando funciones de banca central y con la facultad para emitir moneda que estuviera respaldada por activos comerciales y metales como el oro. El presidente Wilson estuvo de acuerdo con la propuesta en lo general, sin embargo, agregó la figura de una Junta Central, que bajo el control del gobierno, coordinaría los trabajos de los bancos miembros.

Wilson celebró reuniones con los representantes de la Asociación Bancaria Americana ante la Comisión de la Moneda. Pronto el plan atendió solicitudes de los banqueros, entre estas estaban: que sus billetes fueran retirados gradualmente otorgando certidumbre a grandes inversiones de los bancos en bonos respaldados por sus monedas, flexibilidad en la determinación de la tasa de descuento, mayores responsabilidades a los bancos miembros del instituto central y la creación de un Consejo Consultivo Federal integrado por representantes de la comunidad bancaria que sirviera de enlace entre los bancos del instituto y Junta Central. No obstante estos cambios, la comunidad financiera pedía regresar al Plan Aldrich original, con un instituto centralizado y bajo el control de los banqueros.

Después de un intenso cabildeo, la Cámara de Representantes aprobó el Plan Aldrich con una mayoría demócrata. Era el turno ahora del Senado. La minuta fue recibida por la Comisión de Banca de la Colegisladora a cargo del senador republicano Ralph Owen Brewster, antiguo aliado del Plan Aldrich. Esta Cámara decidió abrir al público la discusión sobre la ley que crearía el banco central. Frank A. Valderlip, presidente del National City Bank de Nueva York compareció ante el Comité de Banca del Senado y propuso un nuevo plan bancario y de moneda. El llamado Plan Valderlip contempló: un banco central con un capital suscrito por el público, el gobierno y los bancos nacionales, este banco tendría 12 sucursales en el país, su control descansaría totalmente en manos del gobierno federal y podría emitir moneda respaldada con activos comerciales y $50 \%$ por reservas en oro.

Esta propuesta representó un punto intermedio entre los intereses de izquierda del sector agrícola e industrial y de derecha de los banqueros. A diversos integrantes del ala radical les agradaba la idea del control gubernamental del Plan Valderlip, al igual que a muchos conservadores les simpatizaba porque 
Economía Informa núm. 385 marzo - abril • 2014 - "

mantenía el control en un solo banco. La propuesta Vanderlip fue elegida por un estrecho margen frente al Plan Aldrich reformado (ya aprobado por los representantes). Las versiones que habían sido aprobadas en su momento por el Senado y la Cámara de Representantes tenían ligeras diferencias, mismas que fueron abordadas en los siguientes días mediante la creación de una Conferencia integrada por miembros de ambas Cámaras.

Entre las diferencias destacaba el número de bancos que formarían el instituto central. Lo aprobado por la Cámara de Representantes contemplaba 12, mientras el Senado mandataba máximo 12 y mínimo 8. Los trabajos legislativos de la Conferencia terminaron por aceptar la propuesta del Senado. El 23 de diciembre de 1913 el presidente Wilson firmó la ley que creaba la Reserva Federal (Fed). Sin lugar a dudas, su aprobación había requerido de una enorme negociación política, entre radicales del movimiento progresista y hombres de negocios más prestigiados de la nación. La ley recién aprobada contempló la conformación de un Comité de Organización (en adelante el Comité) integrado por el Secretario del Tesoro, William G. McAdoo, el Secretario de Agricultura, David f. Houston y el Contralor de la Moneda, John Skelton Williams quien fue designado posteriormente.

La tarea del Comité fue elegir las ciudades para asentar los bancos del Fed regionales y dividir el territorio en distritos. La primera ciudad en ser visitada por el Comité fue Nueva York, donde se entrevistó con Morgan, quien señaló que esa ciudad debía tener uno de los bancos regionales, que debía ser el más grande y dominante para que pudiera recibir el reconocimiento de los bancos centrales de Europa. La siguiente ciudad fue Boston, lugar dominado por los partidarios del Plan Aldrich original, donde se apoyaba la existencia de un banco grande en Nueva York y que en Boston hubiera un banco más. El mismo trabajo del Comité continuó en más de 40 ciudades, entre ellas destacaron Washington, Chicago, San Luis, Kansas, Lincoln, Denver, Seattle, Portland, San Francisco, Los Ángeles, El Paso, Austin, Nueva Orleans, Atlanta, Cincinnati y Cleveland.

En abril de 1914 el Comité anunció las doce ciudades sedes de los bancos del Fed: Atlanta, Boston, Chicago, Cleveland, Dallas, Kansas, Minneapolis, Nueva York, Filadelfia, Richmond, San Luis y San Francisco, a su vez los distritos fueron trazados. Los criterios básicos de la elección fueron: la capacidad de los bancos miembros dentro del distrito para poseer un capital mínimo de 4 mil dólares requerido para cada banco del Fed conforme a la ley, las conexiones mercantiles, industriales y financieras existentes en cada distrito, la capacidad del banco del Fed en cada distrito para atender la demanda de los 
negocios que existen en cada localidad, factores geográficos, una red de transporte existente de transporte y comunicación, la población, el área y las actividades de negocios que prevalecían en los distritos (Tallman y Moen, 2006).

El Comité se inclinó porque el número de bancos fuera el máximo posible (12) y que el tamaño de banco del Fed de Nueva York fuera limitado. Aunque, este principio dominó desde un inicio, este banco se limitó al estado de Nueva York con un capital base de 20 millones de dólares, cuatro veces la capitalización de los bancos del Fed de menor tamaño. Para el caso de los bancos del Fed de Atlanta y Minneapolis su capital fue de 5 millones para cada uno. En los hechos fue imposible evitar que el banco del Fed de Nueva York se erigiera como el más grande y dominante entre los demás debido a la enorme cantidad de recursos financieros asentados en esta ciudad. Sin embargo, cabe señalar que resultó ser más pequeño de lo que la comunidad financiera solicitó desde un principio.

Las demás disposiciones debían aplicarse. De acuerdo a la ley los bancos comerciales existentes debían formalizar su relación con el nuevo sistema público, mediante la suscripción de un porcentaje de su capital. Los bancos asociados debían además elegir a quienes los representarían. Para ello, el Comité dividió los bancos privados de cada distrito de acuerdo a su nivel de capitalización en i) los más grandes, ii) los medianos y iii) los más pequeños. Cada grupo debía elegir dos directores por distrito. Un director llamado clase a que representaba a los banqueros y un director clase B, que presentaba liderazgos en sectores tales como la agricultura, la industria, el comercio u otro sector representativo del distrito que no tuviera relación con el sector bancario y financiero. Estos tres grupos debían elegir tres directores clase A y tres directores B a su vez.

La ley también contemplaba los directores clase C, que debían ser elegidos por los miembros de la Junta del Fed a razón de 3 para cada banco miembro del sistema, de entre los cuales uno ocuparía el cargo de presidente y otro de vicepresidente. En el caso de la Junta del Fed, ésta debía ser integrada por 5 miembros propuestos por el presidente y ratificados por el Senado para un periodo de 10 años. A estos 5 se sumarían el Secretario del Tesoro y el Contralor de la Moneda. En mayo de 1914 el Senado recibió los nombres propuestos por el presidente Wilson para conformar dicha Junta, la cual se aprobó con algunos cambios. Finalmente, las nominaciones fueron aprobadas, de entre ellas Charles s. Hamlin fue designado gobernador y Frederic A. Delano vicegobernador de la Junta del Fed. En el plano internacional estaba por comenzar la Primera Guerra Mundial (Roger, 2010). 
Economía Informa núm. 385 marzo - abril • 2014 - "

El año de 1914 el Fed empezó a ejercer sus funciones, entre ellas determinar el control sobre la oferta monetaria. De acuerdo a Friedman (1994) esta función sería "sencilla en principio, porque la oferta monetaria será la que la autoridad quiera que sea; [pero] complicada en la práctica, porque las decisiones de la autoridad monetaria dependerán de numerosos factores, entre éstos figuran las necesidades burocráticas de la autoridad, las creencias y los valores personales de los personajes responsables, las tendencias actuales o supuestas de la economía, las presiones políticas que reciben esas autoridades, y así en inacabable sucesión de detalles". El Fed tuvo la capacidad para descontar las notas (obligaciones) de los banqueros, Eu logró mediante este mecanismo, financiar su participación en la guerra.

Con el conflicto mundial en curso, Benjamín Stron, jefe del Fed de Nueva York de 1914 a 1928 (año en que murió), reconoció que el patrón oro representaba una limitante para el control del crédito. En 1923 dirigió una agresiva acción para detener una recesión mediante una gran compra de bonos de gobierno, lo cual evidenció el poder de las operaciones de mercado abierto para influir en la disponibilidad de crédito en el sistema bancario, después el Fed reconoció este instrumento como una herramienta de su política monetaria. La situación cambio entrada la Gran Depresión, debido a que el Congreso aprobó en 1933 una ley bancaria, mejor conocida como la ley Glass-Steagall, la cual exigió el uso de bonos del gobierno como colaterales para las notas (obligaciones) de la Fed.

La ley también contempló la creación de Federal Deposit Insurance Corporation, lo que colocó a las operaciones de mercado abierto bajo responsabilidad del Fed y pidió que las compañías bancarias conocidas como holding estuvieran bajo su supervisión, una práctica que tendría profundas implicaciones en el futuro. Además como parte de estas reformas, Roosevelt retiró todos los certificados de oro y plata poniendo fin al patrón basado en oro y otros metales. La ley bancaria de 1935 introdujo algunos cambios en la Junta del Fed, con lo cual fue nombrada en adelante como Junta de Gobernadores del Sistema de la Reserva Federal. Esta instancia se conformaría con siete miembros propuestos por el presidente y ratificados por el Senado para un periodo de 14 años. Con la reforma cesó la participación de los titulares del Tesoro y del Contralor de la Moneda en la Junta del Gobernadores del Fed, máximo órgano de decisión de la política monetaria.

Sobre estas modificaciones Friedman (1994) señala que el cambio de nombre de la "Junta de la Reserva Federal" por un nombre más largo como "Junta de Gobernadores del Sistema de la Reserve Federal" se dio por una parte, para elevar el prestigio de este órgano de gobierno, debido a que en la historia 
de los bancos centrales, el director siempre se ha llamado gobernador, ese es el titulo de prestigio. Antes de 1929, los jefes de los doce bancos del Sistema se llamaban gobernadores, atendiendo el deseo de los fundadores, que había sido el establecer un sistema verdaderamente regional y descentralizado. Anterior a la reforma, en la Junta de Gobierno del Fed, sólo el presidente ostentaba el titulo de gobernador, los otros miembros eran sencillamente eso, miembros de la Junta de Gobierno.

Con la reforma a la ley de 1935, los directores de los 12 bancos se llamarían presidentes y los miembros de la Junta de Gobierno del Fed se les nombraría gobernadores, lo cual mostraba en los hechos, la trasferencia real del poder de un sistema regional hacia uno centralizado que lo aglutinaba todo en Washington, distinto a lo que sus fundadores habían logrado. El ejercicio de este poder discrecional que se derivó de la reforma ha sido algunas veces beneficioso, pero más a menudo ha sido perjudicial. El Fed fue testigo de la multiplicación de los precios en más del doble desde que empezó a operar en 1914 hasta la Primera Guerra Mundial. Posteriormente reaccionó de una manera inadecuada, lo que provocó la subsiguiente y grave depresión de 19201921. Tras un breve periodo de estabilidad en los años veinte, sus medidas intensificaron significativamente la contracción de 1929-1933.

En el año de 1951 el entonces presidente, Harry Truman y Secretario del Tesoro, John Snyder, apoyaban el que las tasas de interés bajas permanecieran fijas. El presidente pensó que disminuir el valor de los bonos era su derecho. A pesar de la opinión de Truman y Snyder, la Fed se enfocó en la necesidad de contener las presiones inflacionarias que se agudizaron cuando se estaba desarrollando la Guerra de Corea. Muchos de los que integraban la Junta de Gobernadores incluyendo a Marriner Eccles sugerían que mantener fijas las tasas de interés producía una excesiva expansión monetaria que alimentaba la inflación. Después de un acalorado debate entre el Fed y el Tesoro sobre el control de las tasas de interés y de la política monetaria, su disputa concluyó en un acuerdo conocido como "el acuerdo Tesoro-Fed", el cual eliminó la obligación del banco central para monetizar la deuda del Tesoro. Este acuerdo llegó a ser esencial para definir el rol que juega hoy en día la política monetaria en EU.

\section{Conclusiones}

La creación del Fed en 1913 representó el tercer intento de Eu por contar con un banco central que atendiera los problemas que enfrentaba el sistema bancario y financiero. La aparición del FB y el sB en 1791 y en 1816, respectivamente 
respondieron a la necesidad de contar con bancos nacionales que fungieran como agentes financieros del gobierno. El mayor referente en ese momento era el Banco de Inglaterra, una institución privada. El dotar a la economía de una moneda de circulación nacional generó ventajas y desafíos que se tradujeron en dinamismo económico y crisis financieras por la expansión del crédito. Se experimentó con las operaciones de mercado abierto, logrando que éstas incidieran en el ciclo económico algunas veces con éxito y otras generando graves consecuencias.

Los distintos actores económicos lograron percibir el poder que alcanzó una institución financiera de carácter nacional. Los bancos estatales y regionales vieron minado su actuación frente a la banca nacional. En consecuencia, el FB y el SB no lograron sobrevivir al entorno político nacional que se presentó tan pronto como su renovación fue sometida al Congreso. Casi un siglo tuvo que pasar para la constitución de un nuevo banco nacional. Durante este tiempo, las instituciones bancarias se extendieron de la costa Este a la Oeste y el Centro de Eu rebasando la regulación del estado y creando desordenes bancarios, los pánicos financieros que azotaron a EU durante el siglo XIX y primer cuarto del siglo xx fueron una muestra de ésto. Sin embargo, generaron el ambiente de reflexión para resolver esta situación.

En 13 de diciembre de 1913 se fundó el Fed para dotar a la economía de una moneda elástica y de movilidad de reservas que hiciera frente a las quiebras bancarias que se extendieron a lo largo de EU. El desarrollo de un sistema bancario ligado a la creación de depósitos hizo vulnerable el sistema financiero a cambios bruscos en las preferencias de liquidez de las personas. La respuesta fue la creación de un banco central controlado por el gobierno, descentralizado y con injerencia limitada por parte de los particulares. Su aprobación en el Congreso se concretó después de una intensa y audaz negociación política del gobierno federal. Al final se trató de un experimento único que no tenía referente en ningún lugar del mundo, se trató sin lugar a dudas de una acción pragmática.

La crisis que estalló en 1929 puso a prueba la maquinaria monetaria del gobierno. El desempeño de la política monetaria no logró sacar a EU de la crisis y generó la Gran Depresión. Se relegó entonces la actuación de la política monetaria a cargo del Fed a un segundo plano y se pensó que era "como una cuerda que jalaba pero no empujaba". Las políticas de dinero barato se extendieron en los años que siguieron desatando la Gran Inflación de la década de 1950. La Fed sufrió modificaciones, se dio paso a un sistema centralizado y colaborador del gobierno para financiar el gasto público fundamentalmente 
deficitario. Posteriores desarrollos como: la existencia de una tasa natural de desempleo, expectativas racionales, el problema de inconsistencia dinámica, reglas de política, autonomía de la banca central y blancos de inflación redefinieron el rol de la política monetaria entrada la década de 1980 dando lugar a la Gran Moderación, misma que terminó en 2006 para observar la Gran Recesión que todavía aqueja a EU y diversas partes del mundo.

\section{Bibliografía}

Federal Reserve Bank of Philadelphia (2009), The first bank of the United States, Philadelphia, junio.

Federal Reserve Bank of Philadelphia (2010), The second bank of the United States, Philadelphia, diciembre.

Federal Reserve Bank of Boston (2011), Panic of 1097, Massachusetts.

Friedman, Milton (1994), Money mischief: episodes of monetary history, Harcout Brace and Company Edit.

y Anna J. Schwartz (1963), A monetary history of the United States 1867-1960, Princeton University Press Edit., Nueva Jersey.

y _ (2008), The great contraction 1929-1933, Princeton Clasisc Editions Edit., Nueva Jersey.

Moen, J. y E. Tallman (1999), "Why didn't the United States establish a central bank until after the panic of 1907?”, Working Paper Series 1999-16, Federal Reserve Bank of Atlanta, noviembre.

Roger, T. Johnson (2010), Historical Beginnings the Federal Reserve, Federal Reserve Bank of Boston.

Sylla, R.; R. Wright; D. Cowen (2009), "Alexander Hamilton, central banker: crisis management during the U.S. financial panic of 1792", Business History Review vol. 83, Harvard Business School, Massachusetts.

Tallman, E. y J. Moen (1990), "Lesson from the panic of 1907”, Economic Review, Federal Reserve Bank of Atlanta, mayo.

$\mathrm{y}$

(2006), "Liquidity creation without a lender of last resort: Clearinghouse loan certificates in the banking panic of 1907", Working Paper Series 2006-23, Federal Reserve Bank of Atlanta, noviembre. 